Suay-Hong Gan ${ }^{1}$, Kyi-Win KhinMar ${ }^{1}$, Timothy M. Barkham ${ }^{2}$, Chwee-Kim Koh $^{1}$, Liang Shen ${ }^{3}$, Yee-Tang Wang ${ }^{1}$ and Cynthia B.E. Chee ${ }^{1}$

${ }^{1}$ Tuberculosis Control Unit, Tan Tock Seng Hospital, Singapore. ${ }^{2}$ Dept of Pathology and Laboratory Medicine, Tan Tock Seng Hospital, Singapore. ${ }^{3}$ Biostatistics Unit, National University of Singapore, National University Health System, Singapore.

Correspondence: Suay-Hong Gan, Tuberculosis Control Unit, Tan Tock Seng Hospital, 144 Moulmein Road, 308089, Singapore. E-mail: suay_hong_gan@ttsh.com.sg

Received: Dec 262013 | Accepted after revision: April 022014 | First published online: April 172014

Support statement: This study was funded by the Singapore National Medical Research Council (grant 1004/2005).

Conflict of interest: Disclosures can be found alongside the online version of this article at erj.ersjournals.com

\title{
References
}

World Health Organization. Collaborative framework for care and control of tuberculosis and diabetes. WHO/ HTM/TB/2011.15. Geneva, WHO, 2011.

2 Jeon CY, Murray MB. Diabetes mellitus increases the risk of active tuberculosis: a systematic review of 13 observational studies. PLoS Med 2008; 5: e152.

3 Baker MA, Harries AD, Jeon CY, et al. The impact of diabetes on tuberculosis treatment outcomes: a systematic review. BMC Medicine 2011; 9: 81.

4 Diel R, Loddenkemper R, Zellweger JP, et al. Old ideas to innovate tuberculosis control: preventive treatment to achieve elimination. Eur Respir J 2013; 42: 785-801.

5 D'Ambrosio L, Dara M, Tadolini M, et al. Tuberculosis elimination: theory and practice in Europe. Eur Respir J 2014; 43: 1410-1420.

6 Diel R, Goletti D, Ferrara G, et al. Interferon- $\gamma$ release assays for the diagnosis of latent Mycobacterium tuberculosis infection: a systematic review and meta-analysis. Eur Respir J 2011; 37: 88-99.

7 Diel R, Loddenkemper R, Nienhaus A. Predictive value of interferon- $\gamma$ release assays and tuberculin skin testing for progression from latent TB infection to disease state: a meta-analysis. Chest 2012; 142: 63-75.

8 Migliori GB, Zellweger JP, Abubakar I, et al. European Union standards for tuberculosis care. Eur Respir J 2012; 39: $807-819$.

9 Migliori GB, Sotgiu G, Blasi F, et al. Towards the development of EU/EEA Standards for Tuberculosis Care (ESTC). Eur Respir J 2011; 38: 493-492.

10 Goletti D, Stefania C, Butera O, et al. Accuracy of immunodiagnostic tests for active tuberculosis using single and combined results: a multicentre TBNET-study. PloS One 2008; 3: e3417.

11 Jafari C, Thijsen S, Sotgiu G, et al. Bronchoalveolar lavage enzyme-linked immunospot for a rapid diagnosis of tuberculosis. Am J Respir Crit Care Med 2009; 180: 666-673.

12 Chee CBE, Gan SH, Khinmar KW, et al. Comparison of sensitivities of two commercial gamma interferon release assays for pulmonary tuberculosis. J Clin Microbiol 2008; 46: 1935-1940.

13 Walsh MC, Camerlin AJ, Miles R, et al. The sensitivity of interferon- $\gamma$ release assays is not compromised in tuberculosis patients with diabetes. Int J Tuberc Lung Dis 2011; 15: 179-184.

14 Kabeer BSA, Balambal R, Thomas A, et al. Role of QuantiFERON-TB Gold, interferon gamma inducible preotein10 and tuberculin skin in active tuberculosis diagnosis. PloS One 2010; 5: e9051.

\section{In vitro synergy between linezolid and clarithromycin against Mycobacterium tuberculosis}

To the Editor:

Approximately 3\% of new tuberculosis cases worldwide represent multidrug-resistant tuberculosis (MDRTB) [1]. In these MDR-TB cases, resistance of Mycobacterium tuberculosis to the otherwise effective rifampicin and isoniazid forces clinicians to diverge to other antimicrobial agents. Such treatment options include the World Health Organization (WHO) group 5 drugs linezolid and clarithromycin [1]. Linezolid shows excellent efficacy in the treatment of MDR-TB, but its use is often troubled by adverse events [2-4]. Linezolid has shown in vitro bacteriostatic activity against $M$. tuberculosis and is also effective at achieving culture conversion in drug-resistant cases [5]. In vitro testing revealed that clarithromycin is not very active against $M$. tuberculosis, as the minimal inhibitory concentrations (MICs) are relatively high. Clinical efficacy seems questionable, as MICs, as reported in the literature, are significantly higher than achievable serum peak levels in vivo [6]. Conversely, clarithromycin reaches adequate local concentrations in alveolar cells 
and in epithelial lining fluid, where most mycobacteria reside [7], although lower clarithromycin MICs were observed by the Dutch National Mycobacteria Reference Laboratory (Bilthoven, the Netherlands; unpublished data).

Due to the limited number of new treatment options, optimising existing treatment regimens is a conceivable option. Exploring synergy between tuberculosis drugs might help in improving treatment regimens. A study that investigated several antituberculous drugs, such as isoniazid, rifampicin and/or ethambutol, but not linezolid, revealed in vitro synergistic activity with clarithromycin against M. tuberculosis [8]. In this study, we aimed to investigate the possible in vitro synergy between linezolid and clarithromycin in M. tuberculosis isolates obtained from multidrug-resistant, monoresistant and drugsusceptible tuberculosis cases.

We randomly selected a panel of $24 \mathrm{M}$. tuberculosis isolates from the strain collection of the Tuberculosis Reference Laboratory of the National Institute for Public Health and the Environment (RIVM, Bilthoven, the Netherlands). The selected collection consisted of 13 multidrug-resistant, five drug-sensitive and six monoresistant $M$. tuberculosis isolates. Drug susceptibility testing was performed using two methods: the absolute concentration method (ACM) and a Mycobacteria Growth indicator tube (MGIT) 960 system (Becton Dickinson BV, Breda, the Netherlands) [9, 10].

For the ACM, we used a sterilised Middlebrook 7H10 agar of pH 6.6 supplemented with oleic acid, bovine albumin, dextrose and catalase (both Becton Dickinson and Company, Sparks, MD, USA), and varying concentrations of drugs [9]. The plates were checked for mycobacterial growth after 4, 8, 12, 14, 16, 19 and 21 days. The plates were analysed when the growth in the control well without antituberculous drugs was considered sufficient, i.e. when colonies were clearly visible and countable. At this point, all wells were checked for growth inhibition. Growth inhibition was defined as $<90 \%$ of the colonies of the control well.

Parallel to the ACM, the MGIT 960 system with EpiCenter TB eXiST software (Becton Dickinson and Company, Sparks, MD, USA) was used [10]. Each tube contained $0.8 \mathrm{~mL}$ Bactec MGIT drug susceptibility supplement and $100 \mu \mathrm{L}$ of the appropriate drug solution. Growth was monitored hourly. The tubes were analysed when the growth unit value of the growth control tube, containing a 1:100 dilution of the inocula, reached 400. Growth inhibition was defined as GU value $<100$.

The checkerboard method was used to study in vitro synergy between linezolid and clarithromycin (both Sigma Aldrich, St Louis, MO, USA). Linezolid was added in concentrations between 0 and $0.5 \mu \mathrm{g} \cdot \mathrm{mL}^{-1}$ and clarithromycin with a range of $0-8 \mu \mathrm{g} \cdot \mathrm{mL}^{-1}$, as is shown in figure 1 . We calculated the lowest fractional inhibitory concentration (FIC) to determine synergy as: (MIC of linezolid in combination/MIC of linezolid alone)+(MIC of clarithromycin in combination/MIC of clarithromycin alone). Synergy was defined as a FIC $\leqslant 0.5$, indifference as FIC $>0.5$ to 4 and antagonism as FIC $>4$ [11].

Of the selected M. tuberculosis isolates $(n=24)$, synergy between clarithromycin and linezolid was determined for $74 \%$ by using the MGIT method and in $59 \%$ by using the ACM. The median (interquartile range (IQR)) FIC was $0.37(0.31-0.47)$ using the MGIT and $0.50(0.38-0.75)$ using the ACM. The combination of drugs did not display antagonism in any of the isolates. A median checkerboard composed from all selected M. tuberculosis strains with clarithromycin and linezolid is shown in figure 1 . Synergy was observed in $77 \%$ of the MDR-TB isolates $(n=13)$ using MGIT and in $46 \%$ in the ACM method. Combining clarithromycin and linezolid resulted in a median (IQR) FIC of 0.37 (0.32-0.37) using MGIT and 0.62 (0.375-1.0) using ACM in the MDR-TB isolates.

In conclusion, we observed synergy between clarithromycin and linezolid both with the MGIT and with the ACM method. This finding is in line with a previous observation that clarithromycin displayed synergy with isoniazid, rifampicin and/or ethambutol in M. tuberculosis [8].

Although the underlying mechanism is yet to be elucidated, it has been suggested that disorganisation or disruption of the outer cell wall layer and the cytoplasmic membrane of the cell envelope by either clarithromycin or linezolid may play a role [8]. This disruption might allow easier penetration by the other drug, resulting in the observed in vitro synergy. However, this hypothesis assumes that permeability is normally a limiting factor. Further research studying the underlying mechanism is needed and might also explain the fact that we observed in vitro synergy in the majority, but not all, of the isolates. Although the majority of the isolates in this research displayed in vitro synergy when clarithromycin and linezolid were combined, it would be more interesting to determine or predict which isolates display synergy before applying these drugs in treatment regimens. Indeed, checkerboard experiments have not been validated or widely accepted for tailoring treatment in individual cases, and therefore cut-off values for FIC to deviate from the theoretical cut-off value of 1.0 have been employed [11]. Consequently, the number of isolates displaying synergy might be under- or overestimated. 

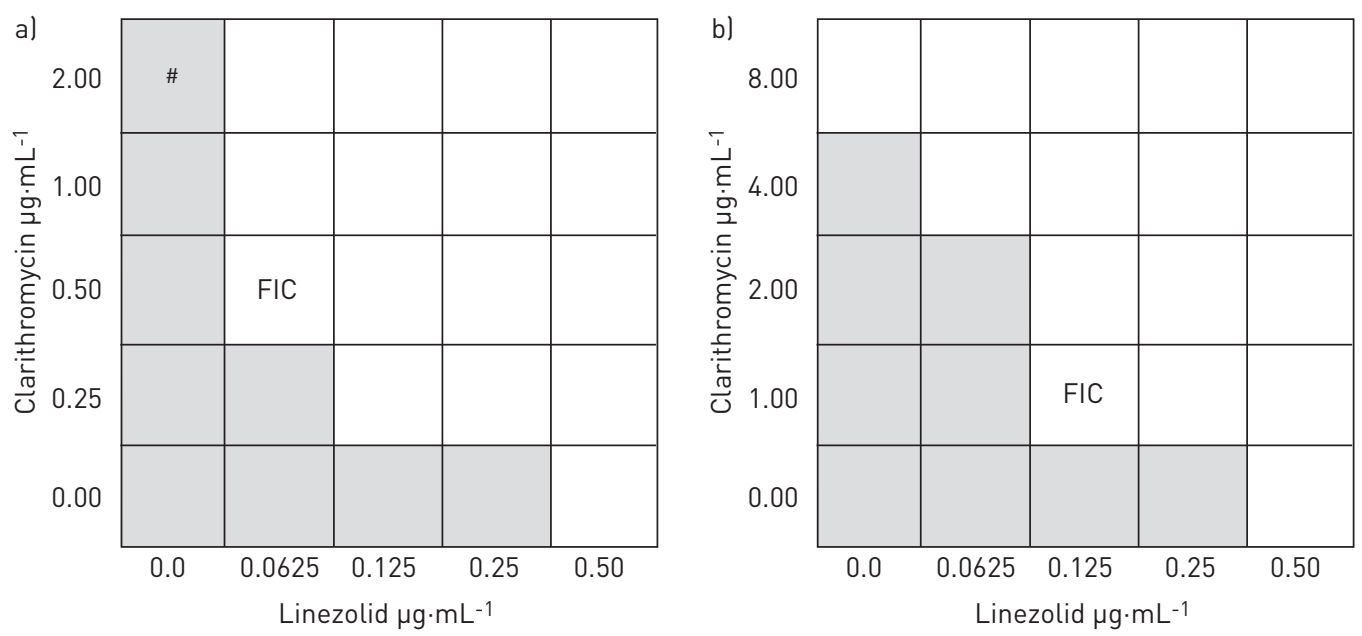

FIGURE 1 Schematic median checkerboard of Mycobacterium tuberculosis growth inhibition with varying concentrations of clarithromycin and/or linezolid using a) Mycobacteria Growth Indicator Tubes $(n=23)$ and b) the absolute concentration method (ACM) $(n=23)$. Each cell represents the median result, i.e. growth or no growth, of all tested isolates. Shaded cells indicate growth and unshaded cells no growth. Lack of growth is defined for a) MGIT as a growth unit value $<100$ at the time the 1:100 growth control tube reaches a value of 400 and for the b) ACM as $<90 \%$ of the colonies compared to the control well. "FIC" indicates the cell representing the lowest fractional inhibitory concentration. For example, the FIC by the ACM (b) is (MIC of linezolid in combination/MIC of linezolid alone)+(MIC of clarithromycin in combination/MIC of clarithromycin alone $)=(0.125 / 0.50)+(1.0 / 8.0)=0.375$. MIC: minimal inhibitory concentration. \#: in order to calculate the FICs, $4 \mu \mathrm{g} \cdot \mathrm{mL}^{-1}$ was used as the MIC of clarithromycin alone.

Previously, we showed that clarithromycin increases linezolid exposure by 44\% in MDR-TB patients [12]. The implications were summarised as: clarithromycin might be used as a booster to increase linezolid exposure, comparable to low-dose ritonavir and protease inhibitors; and the relatively cheap clarithromycin might reduce costs of treatment of the relatively expensive linezolid [13]. The in vitro synergy we observed in this study further strengthens the case for adding clarithromycin as a secondary drug to MDR-TB treatment regimens. The increased drug susceptibility of linezolid and clarithromycin in combination with the increased linezolid exposure might allow for further reduction of linezolid dosage, further reducing costs and adverse events. A prospective evaluation of MDR-TB patients receiving both drugs as a part of their treatment regimen is warranted to investigate efficacy and tolerability in real life. Furthermore, synergy testing should be performed both with other second-line tuberculosis drugs and with new tuberculosis drugs in the pipeline. Especially as the number of new MDR-TB drugs emerging from the pipeline in the next years is expected to be limited, drug resistance should be avoided at all costs. Optimising treatment regimens through use of combinations that show synergy could be one strategy to avoid overextended use of new drugs. This is particularly important when considering the new WHO post-2015 Strategy, which is based on the concept of tuberculosis elimination [14, 15].

To conclude, clarithromycin and linezolid display in vitro synergy in multidrug-resistant M. tuberculosis isolates. Due to the boosting effect with linezolid, low incidence of adverse effects, low costs, observed higher concentrations in lung tissue, and the in vitro synergy with linezolid and other antimicrobial drugs, the role of clarithromycin might become more important in future MDR-TB treatment.

○ @ERSpublications

Clarithromycin and linezolid display in vitro synergy in multidrug-resistant Mycobacterium tuberculosis isolates http://ow.ly/vwf9W

Mathieu S. Bolhuis ${ }^{1}$, Tridia van der Laan ${ }^{2}$, Jos G.W. Kosterink ${ }^{1,3}$, Tjip S. van der Werf ${ }^{4}$, Dick van Soolingen ${ }^{2,5}$ and Jan-Willem C. Alffenaar ${ }^{1}$

${ }^{1}$ University of Groningen, University Medical Center Groningen, Dept of Clinical Pharmacy and Pharmacology, Groningen, The Netherlands. ${ }^{2}$ National Tuberculosis Reference Laboratory, National Institute for Public Health and the Environment (RIVM), Bilthoven, The Netherlands. ${ }^{3}$ University of Groningen, Dept of Pharmacy, Section of Pharmacotherapy and Pharmaceutical Care, Groningen, The Netherlands. ${ }^{4}$ University of Groningen, University Medical Center Groningen, Dept of Internal Medicine and Pulmonary Disease and Tuberculosis, Groningen, The Netherlands. ${ }^{5}$ Radboud University Nijmegen Medical Center, Depts of Clinical Infectious Diseases, Pulmonary Diseases and Medical Microbiology, Nijmegen, The Netherlands. 
Correspondence: Mathieu S. Bolhuis, University of Groningen, University Medical Centre Groningen, Dept of Clinical Pharmacy and Pharmacology, University of Groningen, PO Box 30.001, 9700 RB Groningen, The Netherlands. E-mail: m.s.bolhuis@umcg.nl

Received: March 022014 | Accepted after revision: April 012014 | First published online: May 22014

Conflict of interest: Disclosures can be found alongside the online version of this article at erj.ersjournals.com

\section{References}

1 Falzon D, Jaramillo E, Schunemann HJ, et al. WHO guidelines for the programmatic management of drug-resistant tuberculosis: 2011 update. Eur Respir J 2011; 38: 516-528.

2 De Lorenzo S, Alffenaar JW, Sotgiu G, et al. Efficacy and safety of meropenem-clavulanate added to linezolidcontaining regimens in the treatment of MDR-/XDR-TB. Eur Respir J 2013; 41: 1386-1392.

3 Migliori GB, Eker B, Richardson MD, et al. A retrospective TBNET assessment of linezolid safety, tolerability and efficacy in multidrug-resistant tuberculosis. Eur Respir J 2009; 34: 387-393.

4 Sotgiu G, Centis R, D'Ambrosio L, et al. Efficacy, safety and tolerability of linezolid containing regimens in treating MDR-TB and XDR-TB: systematic review and meta-analysis. Eur Respir J 2012; 40: 1430-1442.

5 Lee M, Lee J, Carroll MW, et al. Linezolid for treatment of chronic extensively drug-resistant tuberculosis. N Engl J Med 2012; 367: 1508-1518.

6 Truffot-Pernot C, Lounis N, Grosset JH, et al. Clarithromycin is inactive against Mycobacterium tuberculosis. Antimicrob Agents Chemother 1995; 39: 2827-2828.

7 Honeybourne D, Kees F, Andrews JM, et al. The levels of clarithromycin and its 14-hydroxy metabolite in the lung. Eur Respir J 1994; 7: 1275-1280.

8 Cavalieri SJ, Biehle JR, Sanders WE Jr. Synergistic activities of clarithromycin and antituberculous drugs against multidrug-resistant Mycobacterium tuberculosis. Antimicrob Agents Chemother 1995; 39: 1542-1545.

9 van Klingeren B, Dessens-Kroon M, van der Laan T, et al. Drug susceptibility testing of Mycobacterium tuberculosis complex by use of a high-throughput, reproducible, absolute concentration method. J Clin Microbiol 2007; 45: $2662-2668$.

10 Alffenaar JW, van der Laan T, Simons S, et al. Susceptibility of clinical Mycobacterium tuberculosis isolates to a potentially less toxic derivate of linezolid, PNU-100480. Antimicrob Agents Chemother 2011; 55: 1287-1289.

11 Odds FC. Synergy, antagonism, and what the chequerboard puts between them. J Antimicrob Chemother 2003; 52: 1.

12 Bolhuis MS, Altena RV, Soolingen DV, et al. Clarithromycin increases linezolid exposure in multidrug-resistant tuberculosis patients. Eur Respir J 2013; 42: 1614-1621.

13 Srivastava S, Peloquin CA, Sotgiu G, et al. Therapeutic drug management: is it the future of multidrug-resistant tuberculosis treatment? Eur Respir J 2013; 42: 1449-1453.

14 Diel R, Loddenkemper R, Zellweger JP, et al. Old ideas to innovate tuberculosis control: preventive treatment to achieve elimination. Eur Respir J 2013; 42: 785-801.

15 D'Ambrosio L, Dara M, Tadolini M, et al. Tuberculosis elimination: theory and practice in Europe, Eur Respir J 2014; 43: 1410-1420.

\section{ERS/WHO Tuberculosis Consilium} assistance with extensively drug-resistant tuberculosis management in a child: case study of compassionate delamanid use

\section{To the Editor:}

The European Respiratory Society (ERS) and the World Health Organization (WHO) Regional Office for Europe implemented a consultation body, the ERS/WHO Tuberculosis (TB) Consilium, in late April 2013 [1-4]. This is a novel, high-priority initiative, as part of the 2012-2013 Presidential plan, to face the growing problem of drug-resistant TB in Europe and globally to support clinicians in managing difficult-to-treat TB cases.

Clinicians are increasingly challenged by difficult-to-treat cases of multidrug-resistant (MDR)-TB (i.e. TB caused by Mycobacterium tuberculosis strains resistant to isoniazid and rifampicin) and extensively drug-resistant (XDR)-TB (i.e. TB caused by MDR-TB strains that are also resistant to at least one fluoroquinolone and one injectable second-line anti-TB drug) [5-8]. MDR/XDR-TB is seriously hampering TB control and elimination in Europe [9-11], as patients require long and expensive regimens with significant adverse effects, while cure rates remain low [7, 8, 12-14]. 